\title{
An Enhanced Algorithm for Image Processing Based Catheter Selection During Right Coronary Angiography
}

\author{
Saeed Alam, Sami Ur Rahman², Adnan Khalii², Fakhre Alam², Khurshid Alam³, Abdul Hadi
}

\begin{abstract}
Background: Image processing based catheter selection is a newly developed procedure in which a catheter is selected based on patient's image data, acquired prior to the intervention. In this technique, the arteries are extracted from the data followed by the computation of geometric parameters for all available catheters.

Objectives: To propose an improved algorithm for estimating geometric parameters such as coronary artery curve angle and length of the coronary artery curve.

Material\& Methods: We propose a new framework to compute the geometric parameters from the patient magnetic resonance / computed tomography images. In order to meet the clinical time constraint, we calculate CACA and CACL in 3D MR cardiac images of the patients.

Results: The method has been tested on seven MR cardiac images provided by Unieklinikum Wrzburg. The results show more accuracy and less computation time as compared to the already developed method.

Conclusion: The proposed algorithm uses an enhanced method for extracting 3D curve to calculate the angle and there by greatly reduces the computation time and improves the accuracy.
\end{abstract}

Keywords: Optimal catheter; Right coronary angiography; Catheter selection; Landmarks; Geometricparameter

\section{This article may be cited as: Alam S, Rahman SU, Khali A, Alam F, Alam K, Hadi A.. An Enhanced Algorithm for Image Processing} Based Catheter Selection During Right Coronary Angiography. J Saidu Med Coll Swat. 2021;11(1):18-23

\section{INTRODUCTION}

Coronary angiography is an examination of the blood vessels of the heart. ${ }^{1}$ It is performed to investigate the presence of any obstruction in the coronary arteries. During angiography, a small incision is made in the upper thigh and a guide wire is inserted into the femoral artery which is threaded towards the aorta. ${ }^{2}$ A catheter is then inserted along the guide wire and is passed through to the coronary arteries. In the next step, contrast material is injected through the catheter and is added to the blood in the small vessels. It renders the blood vessels more opaque and the clear images of the small vessels are obtained..$^{3-4}$

Due to the anatomical variations of the aorta and coronary arteries in different humans, one common catheter cannot be used for all patients. The cardiologists test different catheters for a patient and select the best catheter according to the patient's anatomy ${ }^{5}$. This procedure is time consuming and there is a slight chance of cancer from excessive exposure to radiation. It is also possible that a catheter not matching with the internal anatomy can punctures the artery and causes internal bleeding. ${ }^{6}$ It will be more helpful for the cardiologists to know in advance the optimal

1. Cardiology Unit, Saidu Teaching Hospital, Saidu Medical College, Swat, Pakistan

2. Department of Computer Science and IT, University of Malakand, Pakistan

3. Urology Unit, Nawaz Sharif Kidney Hospital Manglawar, Swat, Pakistan

Correspondence: Dr. Fakhre Alam (PhD Medical Imaging)

Assistant Professor, Department of CS \& IT

University of Malakand, Dir(L), Pakistan

Email.fakhre.uom@gmail.com,Phone:03469004003 catheter before they actually start angiography. Image processing based catheter selection is a newly developed procedure in which a catheter is selected based on patient's image data, acquired prior to the intervention work ${ }^{7}$. In this technique, the arteries are extracted from the MR/CT data and then some geometrical parameters are computed. In the next step, all available catheters are considered and geometric parameters are computed. The patient's image parameters or then compared with the parameters of different catheters and a best catheter is selected based on these computation.

We have developed an enhanced method that performs parameters estimation from patient image data. The method extract coronary artery curve from the image and directly calculate the angle in 3D. On the other hand, the existing method extracts the coronary artery curve and project the curve on the 2D plan which creates ambiguity in the final results. We have enhanced the procedure of extracting the curve from the image by directly finding the CACA, which greatly reduces the computation time. This research work is aimed to provide an efficient and accurate method for the computation of features from the coronary artery for the optimal catheter selection. This research area is novel and unique in its integrity and opens new area of research. It is also expected that our research work will open new dimensions for both researchers and clinicians. 


\section{Related Work}

Catheter selection approaches can be divided into two groups e.g. try and error based andimage processing based.Try and error based approaches, deals with the shape of the aorta and coronary arteries. These approaches help users to select appropriate catheters in angiography. However, there is a little information about patient specific catheter selection in the literature about this approach. Researchers such as Schneider ${ }^{8}$ and $\mathrm{Kirks}^{9}$ discuss the very general cases and the recommended catheters. Kimbiris ${ }^{10}$ focuses only on the anomalous aortic origin of the coronary arteries. Brinkman ${ }^{11}$ published results of a study about the variability of human coronary artery geometry. Myler ${ }^{12}$ worked on right coronaryangioplasty and suggest general guidelines for the selection of proper catheter. On the other hand, similar type of work but for left coronary artery was done by Voda ${ }^{13}$. Amore detail work on catheter selection can be found in the work of Baim, Grossman ${ }^{14}$ and Kern ${ }^{15}$. However, one cannot fully get the idea of best catheter selection from the said work due to the absence of in-depth knowledge in it. Moreover, the work of Baim Grossman and Ken is also based on try and error approach i.e. to obtain best catheter, try more than one catheter of different types till the achievement of appropriate one. In the literature, we can also find some other workon best catheter selection i.e. Sarkar et al. ${ }^{16}$. Sarkar et al practically test different types of catheters on patients. For the experiment, they take 79 catheters of different types and tested it on 24 patients. In the experiment of sarkar et al, an average of three catheters per patient was used to obtained best catheter.

The second category is image processing based approach presented by Rahman et al. ${ }^{6,7}$. In this method, optimal catheter is based on several steps. It estimates the parameters from the selected catheter and from the patient's image data. The geometric parameters that extract from the catheters are catheter's curve angle (CCA) and catheter's curve length (CCL). In the next step, geometric parameters are extracted from the patients MR/CT image of the heart i.e. coronary artery curve angle (CACA) and coronary artery curve length (CACL). After computing parameters from the given patient's image data and from all thecatheters, a formula is applied on the extracted features to select an optimal catheter according to the patient anatomy. The optimal catheter is given as the minimum of all the costs computed for all consideredcatheters $i$.

$$
O C=\arg \min \left\{C C_{i}\right\}
$$

Catheter cost (CC) for every catheter $i$ is computed as follows.

$C C_{i}=a \sqrt{\left(\frac{1}{N_{C L}}\left(C C L_{i}\right)-C A C L\right)^{2}}+b \sqrt{\left(\frac{1}{N_{C A}}\left(C C A_{i}\right)-C A C A\right)^{2}}$

In the above equation, $a$ and $b$ are the weight factors while and are the normalization factors for curve length and curve angle respectively. The equation(2) uses parameters from the considered catheters and from the cardiac image data of the patient. It also assign weighted factors to both parts of the equation.

The existing method of optimal catheter selection ${ }^{6,7}$, calculates the angle between coronary artery and aorta curve from 3D image data on $2 \mathrm{D}$ projected plane. The dimension of $3 \mathrm{D}$ coronary artery curve is reduced to $2 \mathrm{D}$ in order to compare the angle with the catheter's angle which is in $2 D$. The $2 D$ plane is generated from three different points on coronary artery curve and these points are extracted during the coronary artery curve extraction. The projection of 3D curve on 2D plan negatively affects the final computed parameters. There is difference between the structure of projected coronary artery curve on 2D plane and coronary artery curve in 3D image. Therefore, the angle of the coronary artery curve calculated on 2D plane is not always the same as angle in 3D image data. The calculated angle of the coronary artery curve also depends on the projection plane. On two different projectionsplane different results are obtained and there is a large number of possibilities to create a projection plane in the image to project the coronary artery curve.

In this paper, we have developed an improved method that performs computation of coronary artery curve angle (CACA) and coronary artery curve length (CACL) directly in 3D image instead of projecting it on 2D plane. The methodology we have adopted for calculating the different geometric parameters and the obtained results are described in the subsequent sections.

\section{MATERIALAND METHOD}

Obtaining the different geometrical parameters from the patient's MR/CT cardiac image data, our method needs the segmented aorta and coronary arteries from the image data of the patient. In order to segments the aorta and coronary arteries our proposed method can be used with any type of segmentation algorithm.. We have used the algorithm from Flehmann ${ }^{5}$ for the segmentation of 
the aorta and coronary arteries. Parameters estimation from the segmented images are required for the selection of the best fitting catheter for the right coronary artery angiography. The extracted parameters from segmented image are centerline of segmented image, CACA and CACL. The following subsections demonstrate the use of our improved algorithm for computing the CACA and CACL.

\section{Centerline Extraction}

Centerline extraction ${ }^{17-20}$ of coronary arteries and aorta is a challenging problem due to the irregular structure of vessels and uneven heartbeats of the patients. Therefore, more research work need to be done in order to simplify this procedure. We use binary thinning algorithm ${ }^{21}$ in the proposed method that extracts the centerline from the segmented image of the aorta and coronary artery.

\section{Right Coronary Artery Curve Computation}

Coronary artery curve angle (CACA) determines the angle between the aorta and the coronary artery. There are three points namely A, B and C as shown in Figure 1 (b), which calculate the angle between the aorta and right coronary artery.In our method, an axial view search is performed on centerline image for the extraction of CACA angle. Our search for coronary artery curve start from the first slice (abdomen aorta), move towards the top and search for more than two non zero neighbor pixels in the same slice. More than two non zero neighbor pixels in the same slice means that the search pointer is in a place where the RCA exist. We select one pixel with minimum $x$-axis value from the selected pixels, and call this pixel as point $D$ is shown in Figure 1 (a). The minimum $x$-axis criteria try to select a pixel very near to right coronary artery. From point $D$ the search pointer moves toward the RCA and last index of coronary artery is selected as point $C$. In the next step the highest pixel of the aorta i.e. point $E$ are determined. Point $A$ is the midpoint of $D$ and $\mathrm{E}$. Subsequently we start the search pointer from point $A$, move the search pointer downwards and search for more than two non zero neighbor pixels in the same slice. We select a point with maximum $x$-axis from the selected points and call this point as $B$. From point $B$, we move the search pointer towards the tip of CACA $C$, if it finds Point $C$, and then point $B$ is called the branching point of the RCA. If search pointer does not finds point $C$, then search for the same criteria to find branching point $B$.
Figure.1. Right coronary artery curve computation

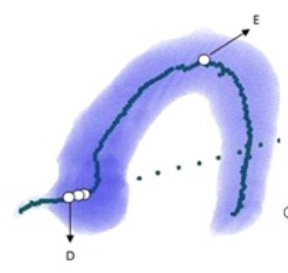

(a)

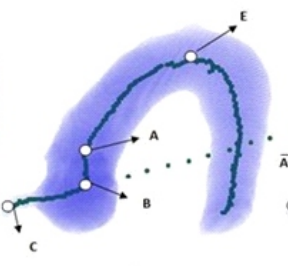

(b)

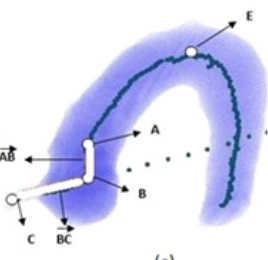

(c)
This method calculated four points on the aorta i.e. point at maximum curvature $E$, half length of the right coronary artery $A$, last index of right coronary artery $C$ and branching point $B$ of aorta and coronary artery. Right coronary artery angle is calculated from points $A, B$ and $C$. The conversion of point $A$ and $B$ to vector and from $B$ and $C$ to vectorare shown in Figure 1 (c). This conversion is performed with the help of equations 3 and 4 . In the equations the coordinates of $A, B$ and $C$ are and $\left[x_{1}, y_{1}, z_{1}\right]\left[x_{2}, y_{2}, z_{2}\right]$ and $\left[x_{3}, y_{3}, z_{3}\right]$ respectively.

$\overrightarrow{A B}=\left[x_{2}-x_{1}, y_{2}-y_{1}, z_{2}-z_{1}\right]$
$\overrightarrow{B C}=\left[x_{3}-x_{2}, y_{3}-y_{2}, z_{3}-z_{2}\right]$

In previous steps, we have obtained two

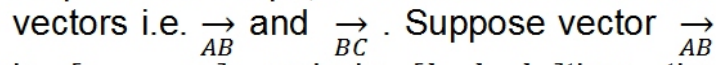
is $\left[a_{1}, a_{2}, a_{3}\right]$ and $\underset{B C}{\rightarrow}$ is $\left[b_{1}, b_{2}, b_{3}\right]$ then the magnitude of $\rightarrow \overrightarrow{A B}$ and $\rightarrow \overrightarrow{B C}$ are obtained with the help of equation 5 and 6 respectively. Magnitudeofvector $\rightarrow \overrightarrow{A B}=r_{1}=\sqrt{a_{1}+a_{2}+a_{3}}$

Magnitudeofvector $\underset{B C}{\rightarrow}=r_{2}=\sqrt{b_{1}+b_{2}+b_{3}}$

Vectors $\rightarrow$ aB and $\rightarrow \overrightarrow{B C}$ are in three dimension, and the angle between two vectors in free space is given by the equation 7 , as follow. $\theta=\csc ^{-1}\left(l_{1} * l_{2}+m_{1} * m_{2}+n_{1} * n_{2}\right)$

Where

$$
l_{1}=\frac{a_{1}}{r_{1}}, m_{1}=\frac{a_{2}}{r_{1}}, n_{1}=\frac{a_{3}}{r_{1}} \text { and }, l_{2}=\frac{b_{1}}{r_{2}}, m_{2}=\frac{b_{2}}{r_{2}}, n_{2}=\frac{b_{3}}{r_{2}}
$$

In the above equation $7, l_{1}, m_{1}, n_{1}$ are the direction cosines of the vector $\overrightarrow{A B}$ and $l_{1}, m_{1}, n_{1}$ are the direction cosines of the vector $\underset{B C}{\rightarrow}$ 


\section{Coronary Artery Curve Length Computation} Coronary artery curve length (CACL) is another important parameter for the selection of optimal catheter before angiography. CACL determine the distance between the branching point $B$ and the tip of the coronary artery $C$ as shown in Figure1 (c). Our proposed method directly calculates CACL in 3D insteadof projecting it on as 2D plan which makes it a better choice to estimate the exact length of coronary artery.

\section{RESULTS}

The experiments were carried out on Intel core i5 with 4GB memory, running under Windows 7 operating system. The algorithm was developed in $\mathrm{C}++$, the Insight registration and segmentation toolkit ${ }^{22}$ and visualization toolkit ${ }^{23}$.

The algorithm was tested on 3D MR cardiac images of seven patients provided by Uniklinikum Wrzburg. The size of the cardiac images was 150 * $150 * 150$. The parameters i.e. coronary artery curve angle and coronary artery curve length from the patients images were extracted using the method already discussed. We have also applied the existing algorithm on the same images which also extract features from them. Our newly developed method was compared with the existing method on the bases of CACA and execution time. The obtain parameters from both algorithms are shown in Table1while execution time to extract parameters from the image are shown in Table 2.
It is shown in Table 1that the proposed method shows different results from the existing method. The proposed method directly calculates the angle in 3D while the existing method calculates the angle on a projected plan. Since, the chance of losing information during the projection of 3D curve on 2D plane is more due to which the existing method produces ambiguous results. On the other hand, our proposed method calculates the angle in 3D which gives more precise and clear results. For example, the coronary artery curve angle estimated for patient 1 in the proposed method and in the existing method are 112.4 and 105.3 respectively. Angle 112.4 is calculated in 3D due to which the original curve remains the same. While the angle 105.5 is estimated on the projection plane due to which the resulting angle shows more change as compared to the original curve.

The time comparison for the execution of both methods is shown in Table 2. The execution time for features extraction in almost every case is less in the proposed method which shows its efficiency. The comparison of elapsed time of the both methods in extraction of parameters is also shown in Figure 2. It isobvious from the Figure that the proposed method is quicker (dotted line) than the existing method(dashed line).

Table 1. Performance evaluation of the proposed algorithm for angle calculation

\begin{tabular}{ccc}
\hline Data Set & $\begin{array}{c}\text { Angle (in degree) } \\
\text { computation in 3D using } \\
\text { proposed method }\end{array}$ & $\begin{array}{c}\text { Angle (in degree) } \\
\text { computation in 2D (existing } \\
\text { method) }\end{array}$ \\
Patient 1 & 112.4 & 105.3 \\
Patient 2 & 153.8 & 134.8 \\
Patient 3 & 123.8 & 120.2 \\
Patient 4 & 94.7 & 100.2 \\
Patient 5 & 115.2 & 123.1 \\
Patient 6 & 93.2 & 100.1 \\
Patient 7 & 158.2 & 152.8 \\
\hline
\end{tabular}

Table 2.Comparing execution time of the proposed algorithm with the existing algorithm

\begin{tabular}{ccc}
\hline Data Set & $\begin{array}{c}\text { Execution time (in second) } \\
\text { of the proposed method }\end{array}$ & $\begin{array}{c}\text { Execution time (in second) } \\
\text { of the Existing method }\end{array}$ \\
Patient 1 & 0.87 & 1.32 \\
Patient 2 & 1.422 & 1.89 \\
Patient 3 & 1.01 & 1.43 \\
Patient 4 & 1.38 & 1.11 \\
Patient 5 & 2.01 & 1.9 \\
Patient 6 & 0.58 & 1.43 \\
Patient 7 & 1.21 & 1.78 \\
\hline
\end{tabular}




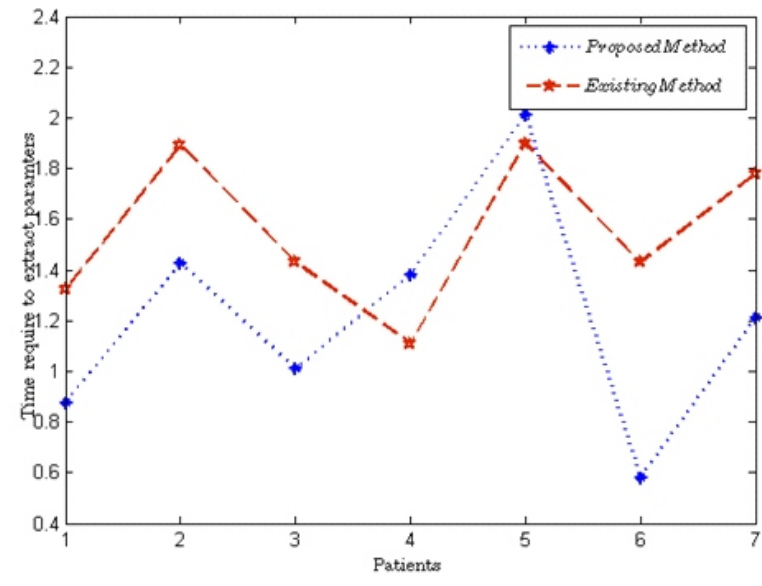

Figure.2. Graphical representation of time require to extract parameters using both methods

\section{DISCUSSION}

The traditional methods of catheter selection (also called trial and error based) are time consuming and risky due to variations in the aorta and coronary arteries in different humans. In the traditional methods, it is not possible to use a common catheter for all patients. The cardiologists test different types of catheters on patient and select an optimal one. It will be more helpful for the cardiologists if they select an optimal catheter (according to patient anatomy) prior to coronary artery angiography. Image processing based patient specific catheter selection is a new and emerging field in the area which can overcome all these issues. The selection of patient specific catheter before coronary angiography not only reduces the exposure time to radiation but also the possible risk of artery punctures and internal bleeding.

We have developed an improved algorithm for calculating the CACA and CACL. The algorithm uses an enhanced method for extracting 3D curve to calculate the angle and there by greatly reduces the computation time and improves the accuracy. The main difference between our method and the previous method is that they project the coronary artery on a 2D plane, while in our method the curve is directly computed in 3D. Therefore, avoid the errors in computation caused by the projection of 3D curve on a 2D plane. In the existing method, coronaryartery curve is computed following the centerline, which needs extra computation to extract the curve. On the other hand, the proposed method search for more than two connected pixels in the same slice and then finds the rest of coronary artery curve using centerline. This feature of the proposed method greatly reduces the computation time to extract the parameters from the patient image.
The existing method fixes the coronary artery curve in thirty slices, while the proposed method does not fixes the curve in 30 slices. Because of this feature the obtained curve is exactly half of the coronary artery curve due to which the accurate angle between aorta and coronary is estimated. The selection of the points and parameters estimation does not need any interaction and estimate the parameters automatically. This parameter estimation serves as a basis for the recommendation of the best fitting catheter to be used during angiography.

\section{CONCLUSION}

In this paper, we have presented an algorithm which automatically extract coronary artery curve from the image and calculate the angle in 3D. The proposed algorithm uses an enhanced image processing based method for extracting 3D curve to calculate the angle and there by greatly reduces the computation time and improves the accuracy.

\section{REFERENCES}

1. Morteza, T, Ashraf, S, Brener, S.J. Risks and complications of coronary angiography: a comprehensive review. Global journal of health science. 2012;4(1): 65-93.

2. Arnett DK, Blumenthal RS, Albert MA, Buroker AB, Goldberger ZD, Hahn EJ, et al. 2019 ACC/AHA guideline on the primary prevention of cardiovascular disease: executive summary: a report of the American College of Cardiology/American Heart Association Task Force on Clinical Practice Guidelines. Journal of the American College of Cardiology. 2019 Sep 2; 74(10):1376-414.

3. Saw J. Coronary angiogram classification of spontaneous coronary artery dissection. Catheterization and Cardiovascular Interventions. 2014 Dec 1;84(7):1115-22.

4. Gupta S, Meyersohn NM, Wood MJ, Steigner ML, Blankstein R, Ghoshhajra BB, et al. Role of Coronary CT Angiography in Spontaneous Coronary Artery Dissection. Radiology: Cardiothoracic Imaging. 2020 Nov 19; 2(6):e200364.

5. Eugen, F, Rahman, S, Wesarg, S, Voelker, W. Towards patient specific catheter selection: computation of aortic geometry based on fused mri data. International Conference on Functional Imaging and Modeling of the Heart. 2011; 6(1): 145-152.

6. Rahman, S, Wesarg, S, Völker, W. Patient specific optimal catheter selection for right coronary artery. Medical Imaging 2011: Visualization, Image-Guided Procedures, and Modeling. 2011; 7964(1): 796412796418.

7. Rahman, S, Wesarg, S, Völker, W. Patient specific optimal catheter selection for the left coronary artery. Gl-Jahrestagung. 2011; 446-52.

8. Gallagher J. Endovascular skills: Guidewire and catheter skills for endovascular surgery. Journal of Vascular Surgery. 2004 Sep 1; 40(3):597-604. 
9. Kirks DR, Fitz CR, Harwood-Nash DC. Pediatric abdominal angiography: Practical guide to catheter selection, flow rates, and contrast dosage. Pediatric radiology. 1976 Mar 1;5(1):19-23.

10. Kimbiris DE, Iskandrian AS, Segal BL, Bemis CE. Anomalous aortic origin of coronary arteries. Circulation. 1978 Oct; 58(4):606-15.

11. Brinkman AM, Baker PB, Newman WP, Vigorito R, Friedman $\mathrm{MH}$. Variability of human coronary artery geometry: an angiographic study of the left anterior descending arteries of 30 autopsy hearts. Annals of biomedical engineering. 1994 Jan 1; 22(1):34-44.

12. Myler RK, Boucher RA, Cumberland DC, Stertzer SH. Guiding catheter selection for right coronary artery angioplasty. Catheterization and cardiovascular diagnosis. 1990 Jan;19(1):58-67.

13. Voda J. Long-tip guiding catheter: Successful and safe for left coronary artery angioplasty. Catheterization and cardiovascular diagnosis. 1992 Nov;27(3):234-42.

14. Baim DS. Angioplasty and General Coronary Intervention. Grossman's Cardiac Catheterization, Angiography, and Intervention. 2006:433.

15. Sorajja P, Lim MJ, Kern MJ. Cardiac Catheterization Handbook E-Book. Elsevier Health Sciences; 2019 Oct 31.

16. Sarkar K, Sharma SK, Kini AS. Catheter selection for coronary angiography and intervention in anomalous right coronary arteries. Journal of interventional cardiology. 2009 Jun;22(3):234-9.

17. Yang G, Kitslaar P, Frenay M, Broersen A, Boogers MJ, Bax JJ, et al . Automatic centerline extraction of coronary arteries in coronary computed tomographic angiography. The international journal of cardiovascular imaging. 2012 Apr 1;28(4):921-33

18. Hernandez-Vela A, Gatta C, Escalera S, Igual L, Martin-Yuste V, Sabate M, et al. Accurate coronary centerline extraction, caliber estimation, and catheter detection in angiographies. IEEE Transactions on Information Technology in Biomedicine. 2012 Sep 28;16(6):1332-40.

19. Li Z, Zhang Y, Liu G, Shao H, Li W, Tang X. A robust coronary artery identification and centerline extraction method in angiographies. Biomedical Signal Processing and Control. 2015 Feb 1; 16: 1-8.

20. Kumar RP, Albregtsen F, Reimers M, Edwin B, Langø $T$, Elle OJ. Three-dimensional blood vessel segmentation and centerline extraction based on twodimensional cross-section analysis. Annals of biomedical engineering. 2015 May 1; 43(5):1223-34.

21. Homann $H$. Implementation of a $3 D$ thinning algorithm. Insight Journal. 2007 Oct 12; 421.

22. Schroeder W, Ng L, Cates J. The ITK Software Guide Second Edition Updated for ITK version 2.4. FEBS Lett. 2005 Nov 21; 525:53-8.

23. Hanwell MD, Martin KM, Chaudhary A, Avila LS. The Visualization Toolkit (VTK): Rewriting the rendering code for modern graphics cards. Software X. 2015 Sep 1;1:9-12.
DATA SHARING STATEMENT: The data that support the findings of this study are available on request from the corresponding author. The data are not publicly available due to privacy or ethical restrictions.

CONFLICT OF INTEREST: Authors declare no conflict of interest.

GRANTED SUPPORT AND FINANCIAL DISCLOSURE:
Nil

\section{AUTHOR'S CONTRIBUTION}

Following authors have made substantial contributions to the manuscript as under

Alam S:
Rehman SU:
of data, statistical analysis
Writing of manuscript, critical review of
manuscript
Khalil A, Alam F: Analysis and interpretation of data,
statistical analysis
Dlam K, Hadi A: Data collection, bibliography
inthors agree to be accountable for all aspects of the work
in ensuring that questions related to the accuracy or
integrity of any part of the work are appropriately
investigated and resolved.

